Document downloaded from:

http://hdl.handle.net/10251/52823

This paper must be cited as:

Liu, X.; Lingling, W.; Benítez López, J. (2011). On linear combinations of generalized involutive matrices. Linear and Multilinear Algebra. 59(11):1221-1236.

doi:10.1080/03081087.2010.496111.

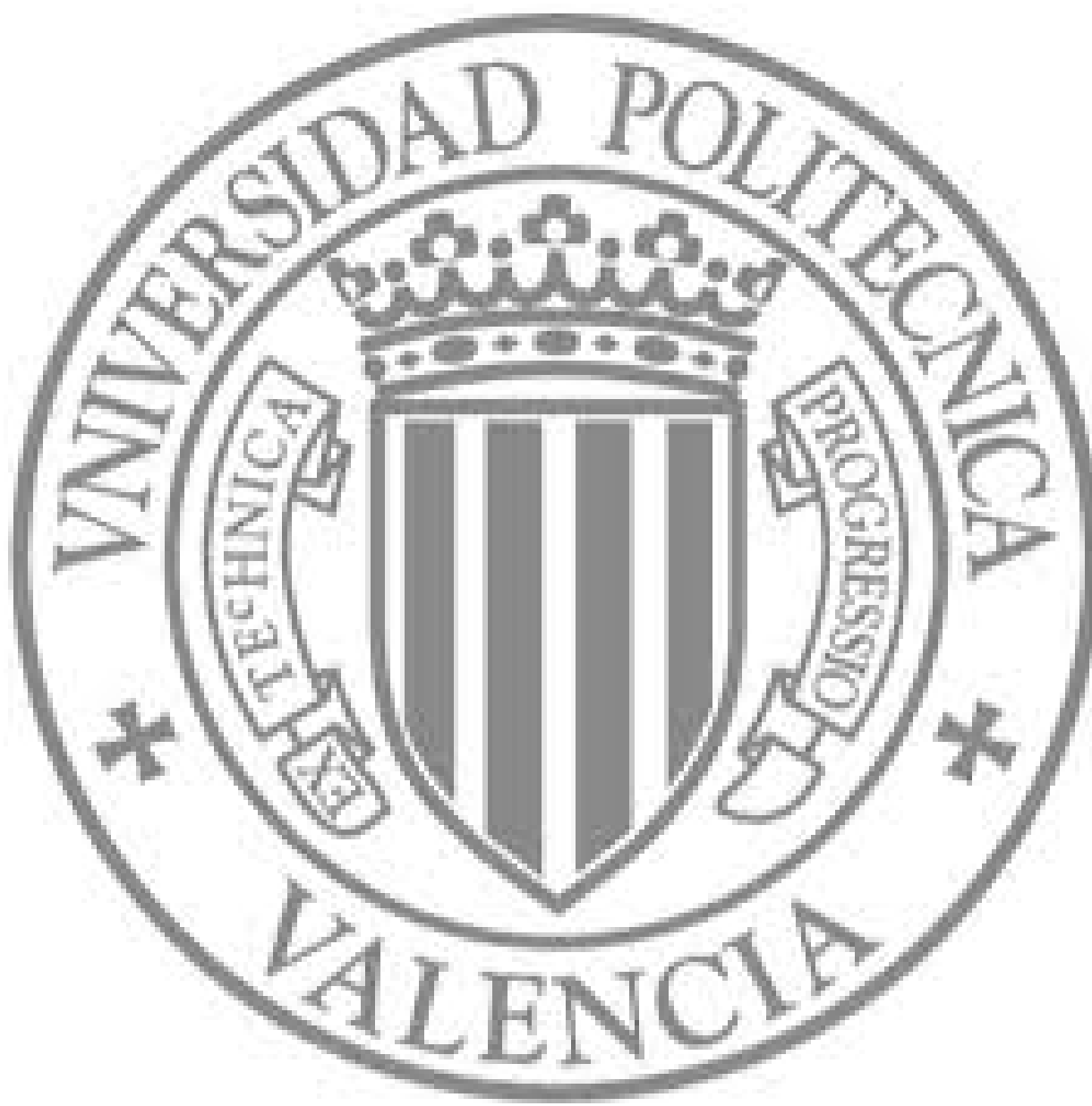

The final publication is available at

http://dx.doi.org/10.1080/03081087.2010.496111

Copyright Taylor \& Francis 


\title{
On linear combinations of generalized involutive matrices.
}

\author{
Xiaoji Liu * $\quad$ Lingling $\mathrm{Wu}^{\dagger} \quad$ Julio Benítez ${ }^{\ddagger}$
}

\begin{abstract}
Let $X^{\dagger}$ denotes the Moore-Penrose pseudoinverse of a matrix $X$. We study a number of situations when $(a A+b B)^{\dagger}=a A+b B$ provided $a, b \in \mathbb{C} \backslash\{0\}$ and $A, B$ are $n \times n$ complex matrices such that $A^{\dagger}=A$ and $B^{\dagger}=B$.
\end{abstract}

AMS Classification: 15A57; 15A09

Keywords: Generalized involutive matrices; CS decomposition; EP matrices.

\section{Introduction}

Let $\mathbb{C}_{m, n}$ be the set of $m \times n$ complex matrices. The symbol $K^{*}$ will denote the conjugate transpose of $K \in \mathbb{C}_{m, n}$. Further, $K^{\dagger}$ will stand for the Moore-Penrose inverse of $K \in \mathbb{C}_{m, n}$, i.e., the unique matrix $K^{\dagger} \in \mathbb{C}_{n, m}$ satisfying the following four conditions:

$$
K K^{\dagger} K=K, \quad K^{\dagger} K K^{\dagger}=K^{\dagger}, \quad\left(K K^{\dagger}\right)^{*}=K K^{\dagger}, \quad\left(K^{\dagger} K\right)^{*}=K^{\dagger} K .
$$

Moreover, $\mathbb{C}_{n}^{\mathrm{EP}}$ and $\mathbb{C}_{n}^{\mathrm{U}}$ will mean the subsets of $\mathbb{C}_{n, n}$ consisting of EP and unitary matrices, respectively, i.e., $\mathbb{C}_{n}^{\mathrm{EP}}=\left\{K \in \mathbb{C}_{n, n}: K K^{\dagger}=K^{\dagger} K\right\}$ and $\mathbb{C}_{n}^{U}=\left\{U \in \mathbb{C}_{n, n}: U^{*}=U^{-1}\right\}$. The symbol $I$ will stand for the identity matrix of suitable size. An important class of matrices will appear throughout the paper: the orthogonal projectors: A square matrix $P$ is an orthogonal projector if it satisfies $P^{2}=P=P^{*}$. It is evident that $P P^{\dagger}$ and $P^{\dagger} P$ are orthogonal projectors for any matrix $P$.

The concept of a generalized involutive element of a $C^{*}$-algebra $\mathcal{A}$ was introduced by Boasso in 2006 in [8]. In a $C^{*}$-algebra $\mathcal{A}$, for a given element $a \in \mathcal{A}$, we can define the MoorePenrose inverse of $a$ as an element $a^{\dagger} \in \mathcal{A}$ satisfying similar relations as in (1). But in the more general setting of $C^{*}$-algebras, it may happen that an element does not have a Moore-Penrose inverse (if the Moore-Penrose inverse exists, it is unique). An element $a$ of a $C^{*}$-algebra having a Moore-Penrose inverse will be called generalized involutive if $a=a^{\dagger}$. Boasso called this

\footnotetext{
${ }^{*}$ Corresponding author. College of Mathematics and Computer Science, Guangxi University for Nationalities, Nanning 530006, P.R. China. xiaojiliu72@yahoo.com.cn

${ }^{\dagger}$ College of Mathematics and Computer Science, Guangxi University for Nationalities, Nanning 530006, P.R. China. wulingling-1982@163.com

${ }^{\ddagger}$ Departamento de Matemática Aplicada, Universidad Politécnica de Valencia, Camino de Vera s/n. 46022, Valencia, Spain. jbenitez@mat.upv.es
} 
class of elements as "Moore-Penrose Hermitian", but a more suitable expression seems to be "generalized involutive" since a matrix equals to its inverse is called involutive.

Obviously, the aforementioned definition is meaningful in the $\mathbb{C}_{n, n}$ setting. We shall denote by $\mathbb{C}_{n}^{\mathrm{Gl}}$ the subset of $\mathbb{C}_{n, n}$ consisting of matrices $A$ such that $A=A^{\dagger}$. It should be obvious that $A=A^{\dagger}$ if and only if $A^{3}=A$ (a matrix $X$ such that $X^{3}=X$ is customary named tripotent) and $\left(A^{2}\right)^{*}=A^{2}$. It is also evident that $\mathbb{C}_{n}^{\mathrm{Gl}} \subset \mathbb{C}_{n}^{\mathrm{EP}}$. In fact, one has that $A \in \mathbb{C}_{n}^{\mathrm{Gl}}$ if and only if $A \in \mathbb{C}_{n}^{\mathrm{EP}}$ and $A^{3}=A$. The "if" implication is trivial and the "only if" is true in view of the theorem given below which constitutes part (i) $\Leftrightarrow$ (iv) of Theorem 4.3.1 in [9].

Theorem 1. Let $K \in \mathbb{C}_{n, n}$ be of rank $r$. Then $K \in \mathbb{C}_{n}^{\mathrm{EP}}$ if and only if there exists $U \in \mathbb{C}_{n}^{\mathrm{U}}$ and nonsingular $K_{1} \in \mathbb{C}_{r, r}$, such that $K=U\left(K_{1} \oplus 0\right) U^{*}$.

If $P$ is generalized involutive, then $P^{2}$ is an orthogonal projector. Also, any orthogonal projector is a generalized involutive matrix, which shows that the set composed of generalized involutive matrices generalizes the set composed of orthogonal projectors. This inclusion is proper because $-1 \in \mathbb{C}_{1,1}$ is a generalized involutive matrix and is not an orthogonal projector. Also, any tripotent and Hermitian matrix is generalized involutive, but the following example shows that the reverse inclusion does not hold: let

$$
A=\left[\begin{array}{ll}
1 & -2 \\
0 & -1
\end{array}\right] \text {. }
$$

Trivially we have $A^{3}=A$ and $A^{2}=I$, which shows that $A \in \mathbb{C}_{n}^{\mathrm{Gl}}$, and however $A$ is not Hermitian. Let us remark that if we impose a condition concerning the rank, any generalized involutive matrix is tripotent and "almost" Hermitian: precisely we have the following result:

Theorem 2. Let $A \in \mathbb{C}_{n, n}$ have rank 1. Then $A \in \mathbb{C}_{n}^{\mathrm{Gl}}$ if and only if there exists $\mathbf{u} \in \mathbb{C}_{n, 1}$ such that $\mathbf{u}^{*} \mathbf{u}=1$ and $A \in\left\{\mathbf{u u}^{*},-\mathbf{u u}^{*}\right\}$.

Proof. Let $\mathbf{u} \in \mathbb{C}_{n, 1}$ such that $\mathbf{u}^{*} \mathbf{u}=1$. Is trivial to prove that $\mathbf{u u}^{*}$ and $-\mathbf{u u}^{*}$ are generalized involutive matrices.

Assume that $A \in \mathbb{C}_{n}^{\mathrm{GI}}$. By using that $A \in \mathbb{C}_{n}^{\mathrm{EP}}$ and Theorem 1 , there exists a unitary matrix $U \in \mathbb{C}_{n, n}$ and $\lambda \in \mathbb{C} \backslash\{0\}$ such that $A=U(\lambda \oplus 0) U^{*}$. Since $A=A^{\dagger}$ we obtain $\lambda=\lambda^{-1}$, i.e., $\lambda \in\{1,-1\}$. Let us partition $U$ as follows: $U=[\mathbf{u} V]$, being $\mathbf{u} \in \mathbb{C}_{n, 1}$ and $V \in \mathbb{C}_{n, n-1}$. From $A=U(\lambda \oplus 0) U^{*}$ we get $A=\lambda \mathbf{u u}^{*}$.

By mimicking the proof of Theorem 2 we can establish the following characterization of the generalized involutive matrices.

Theorem 3. Let $A \in \mathbb{C}_{n, n}$ have rank $r$. Then $A \in \mathbb{C}_{n}^{\mathrm{Gl}}$ if and only if there exists $U \in \mathbb{C}_{n, r}$ and $K \in \mathbb{C}_{r, r}$ such that the columns of $U$ are orthonormal, $K^{2}=I$, and $A=U K U^{*}$.

This result is another justification of the name "generalized involutive matrices". Let us remark that Theorem 3 provides us concrete examples of generalized involutive matrices.

The generalized involutive matrices are strongly related to the set of hypergeneralized projectors defined as $\left\{K \in \mathbb{C}_{n, n}: K^{2}=K^{\dagger}\right\}$ introduced by Groß and Trenkler in [12]. Subsequently Baksalary et al. in [1] derived some properties of the hypergeneralized projectors. 
One can see [18] for a spectral characterization of the hypergeneralized projectors (and also for $\left.\mathbb{C}_{n}^{\mathrm{Gl}}\right)$.

A challenging question concerning to $\mathbb{C}_{n}^{\mathrm{Gl}}$ is: when is a linear combination of the form

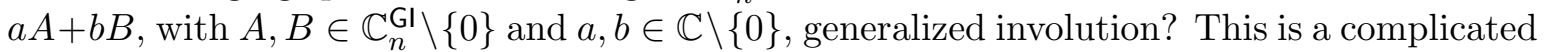
problem since the derivation of necessary conditions for $(a A+b B)^{\dagger}=a A+b B$ may depend on the formula for the Moore-Penrose inverse of a sum of matrices (see [14]). In this paper, we provide a solution of this problem corresponding to many situations by using block matrices.

Let us remark that a related problem was solved in [3], where it was studied when a linear combination $a A+b B$ is a hypergeneralized projection provided $A, B \in \mathbb{C}_{n, n}$ are hypergeneralized projections and $a, b \in \mathbb{C} \backslash\{0\}$, only under the assumption $A B=B A$. It is worthy that we study more situations besides the commutativity.

Let us mention some applications where the generalized involutive matrices appear.

The called set inclusion matrices (see [4] for the definition) arise in many combinatorial problems. In [4] (the final example), the author constructs an explicit set inclusion matrix $W$ such that $W=W^{\dagger}$.

In [19] (see also [17, pg. 167]) it was characterized the real symmetric idempotent matrices. Among the list of equivalent conditions one can se that $A$ is a real symmetric idempotent matrix if and only if $A$ is idempotent and $A=A^{\dagger}$.

Let $\mathbf{x}$ be an $d \times 1$ real random vector having the multivariate normal distribution $N_{d}(\boldsymbol{\mu}, \boldsymbol{\Sigma})$, where $\boldsymbol{\Sigma}$ is nonnegative definite of rank $r$ (with $r<d$ ). Then ([16, Theorem 9.12]) $\mathbf{y}=\mathbf{x}^{\prime} \boldsymbol{\Sigma}^{\dagger} \mathbf{x}$ has a chi-squared distribution with noncentrality parameter $\boldsymbol{\delta}=\boldsymbol{\mu}^{\prime} \boldsymbol{\Sigma} \boldsymbol{\mu}$, where the superscript $'$ denotes the matrix transposition. If $\boldsymbol{\Sigma}^{\dagger}=\boldsymbol{\Sigma}$, then the quadratic form of $\mathbf{y}$ and $\boldsymbol{\delta}$ are the same.

\section{Main results}

This following simple observation will be useful: given $A \in \mathbb{C}_{n}^{\mathrm{Gl}} \backslash\{0\}$ and $a \in \mathbb{C} \backslash\{0\}$, then $a A \in \mathbb{C}_{n}^{\mathrm{Gl}}$ if and only if $a \in\{-1,1\}$. In what follows, we assume that $a, b \in \mathbb{C}$ are nonzero and $A, B \in \mathbb{C}_{n}^{\mathrm{Gl}}$ are linearly independent matrices, since otherwise such situations lead to trivial characterizations.

The following result concerning two commuting EP matrices was proved in [5, Corollary 3.9].

Lemma 1. Let $A, B \in \mathbb{C}_{n, n}$ be two EP matrices such that $A B=B A$. Then there exists $U \in \mathbb{C}_{n}^{\mathrm{U}}$ satisfying

$$
A=U\left(A_{1} \oplus A_{2} \oplus 0 \oplus 0\right) U^{*}, \quad B=U\left(B_{1} \oplus 0 \oplus B_{2} \oplus 0\right) U^{*},
$$

where $A_{1}, A_{2}, B_{1}$, and $B_{2}$ are nonsingular matrices and $A_{1} B_{1}=B_{1} A_{1}$.

Next result constitutes the solution to the problem posed in the introduction when the involved matrices commute. 
Theorem 4. Let $A, B \in \mathbb{C}_{n}^{\mathrm{Gl}}$ be linearly independent satisfying $A B=B A$ and $a, b \in \mathbb{C} \backslash\{0\}$. Then $a A+b B$ belongs to $\mathbb{C}_{n}^{\mathrm{Gl}}$ if and only if any of the following sets of conditions holds:

(a) $(a, b) \in\{(1,-1),(-1,1)\}$ and $A^{2} B=A B^{2}$.

(b) $(a, b) \in\{(1,-2),(-1,2)\}$ and $A B=B^{2}$.

(c) $(a, b) \in\{(2,-1),(-2,1)\}$ and $A B=A^{2}$.

(d) $(a, b) \in\{(1,1),(-1,-1)\}$ and $A^{2} B=-A B^{2}$.

(e) $(a, b) \in\{(1,2),(-1,-2)\}$ and $A B=-B^{2}$.

(f) $(a, b) \in\{(2,1),(-2,-1)\}$ and $A B=-A^{2}$.

(g) $(a, b) \in\left\{\left(\frac{1}{2}, \frac{1}{2}\right),\left(\frac{1}{2},-\frac{1}{2}\right),\left(-\frac{1}{2}, \frac{1}{2}\right),\left(-\frac{1}{2},-\frac{1}{2}\right)\right\}, A^{2}=B^{2}$, and $A B$ is Hermitian.

Proof. Since $A$ and $B$ are two commuting EP matrices, by Lemma 1, there exists $U \in \mathbb{C}_{n}^{U}$ such that $A$ and $B$ are represented as in (2). Moreover, from $A^{3}=A, B^{3}=B$, and the nonsingularity of $A_{1}, A_{2}, B_{1}$, and $B_{2}$ we deduce

$$
A_{1}^{2}=B_{1}^{2}=I, \quad A_{2}^{2}=I, \quad B_{2}^{2}=I .
$$

First we suppose that $a A+b B \in \mathbb{C}_{n}^{\mathrm{Gl}}$. Since $(a A+b B)^{3}=a A+b B, A^{3}=A, B^{3}=B$, and $A B=B A$, we can apply the main result in [2], obtaining seven conditions.

(i) $(a, b) \in\{(1,-1),(-1,1)\}$ and $A^{2} B=A B^{2}$.

(ii) $(a, b) \in\{(1,-2),(-1,2)\}$ and $A^{2} B=B=A B^{2}$.

(iii) $(a, b) \in\{(2,-1),(-2,1)\}$ and $A^{2} B=A=A B^{2}$.

(iv) $(a, b) \in\{(1,1),(-1,-1)\}$ and $A^{2} B=-A B^{2}$.

(v) $(a, b) \in\{(1,2),(-1,-2)\}$ and $A^{2} B=B=-A B^{2}$.

(vi) $(a, b) \in\{(2,1),(-2,-1)\}$ and $A^{2} B=-A=-A B^{2}$.

(vii) $(a, b) \in\left\{\left(\frac{1}{2}, \frac{1}{2}\right),\left(\frac{1}{2},-\frac{1}{2}\right),\left(-\frac{1}{2}, \frac{1}{2}\right),\left(-\frac{1}{2},-\frac{1}{2}\right)\right\}, A^{2} B=B, A B^{2}=A$.

We shall prove that condition (ii) leads to (b) and condition (vii) together the hermitianess of $(a A+b B)^{2}$ leads to $(\mathrm{g})$, respectively, since the remaining ones or are trivial or can be proven similarly.

Suppose (ii) holds. If we employ (2) and (3) we get $A_{1}=B_{1}$ and the third summand in the representation (2) is absent because $B_{2}$ is nonsingular. Hence $A B=B^{2}$.

Suppose (vii) holds. After using (2), (3), and the nonsingularity of $A_{2}$ and $B_{2}$ we obtain that the second and the third summand in the representation (2) are absent. Therefore, $A=U\left(A_{1} \oplus 0\right) U^{*}$ and $B=U\left(B_{1} \oplus 0\right) U^{*}$, and thus $A^{2}=B^{2}$. Moreover, since $a A+b B \in \mathbb{C}_{n}^{\mathrm{Gl}}$ 
then $(a A+b B)^{2}$ is Hermitian. Having in mind that $a, b \in \mathbb{R}$ and $A^{2}, B^{2}$ are Hermitian, we get that $A B$ is also Hermitian.

Now, we shall prove the sufficiency of the conditions (a)-(g) listed in the theorem, i.e., we shall see that any of the conditions (a) $-(\mathrm{g})$ implies $(a A+b B)^{3}=a A+b B$ and the hermitianess of $(a A+b B)^{2}$.

In order to prove the tripotency of $a A+b B$, in view of the main result of [2], it is enough to prove that conditions (a) $-(\mathrm{g})$ lead to conditions (i) - (vii), respectively. We only prove (b) $\Rightarrow$ (ii) and $(\mathrm{g}) \Rightarrow($ vii) since the others are trivial or similar. Suppose that (b) holds. Now,

$$
A^{2} B=A(A B)=A B^{2}=(A B) B=B^{3}=B .
$$

Suppose that (g) holds. Now we have $A^{2} B=B^{2} B=B^{3}=B$, and in a similar way, $B^{2} A=A$.

Now, we shall prove that $(a A+b B)^{2}$ is Hermitian. Since $a, b \in \mathbb{R}$ and $A^{2}, B^{2}$ are Hermitian, it is enough to prove that $A B$ is Hermitian. Obviously, the only non trivial conditions are $(\mathrm{a}) \Rightarrow$ " $A B$ is Hermitian" and $(\mathrm{d}) \Rightarrow$ " $A B$ is Hermitian". We shall only prove the first of them: Suppose that (a) holds. Using relations (2) and (3) we get $A_{1}=B_{1}$, and having in mind relations (3) again we get $A B=U(I \oplus 0 \oplus 0 \oplus 0) U^{*}$, which is clearly Hermitian. The proof is completed.

In next lines, we will give examples showing that conditions (a)-(g) of Theorem 4 are not void.

(a) $A=\operatorname{diag}\left(\lambda_{1}, \lambda_{2}, 0\right), B=\operatorname{diag}\left(\lambda_{1}, 0, \lambda_{3}\right), \lambda_{1}, \lambda_{2}, \lambda_{3} \in\{-1,1\}$.

(b) $A=\operatorname{diag}\left(\lambda_{1}, \lambda_{2}\right), B=\operatorname{diag}\left(\lambda_{1}, 0\right), \lambda_{1}, \lambda_{2} \in\{-1,1\}$.

(c) $A=\operatorname{diag}\left(\lambda_{1}, 0\right), B=\operatorname{diag}\left(\lambda_{1}, \lambda_{2}\right), \lambda_{1}, \lambda_{2} \in\{-1,1\}$.

(d) $A=\operatorname{diag}\left(\lambda_{1}, \lambda_{2}, 0\right), B=\operatorname{diag}\left(-\lambda_{1}, 0, \lambda_{3}\right), \lambda_{1}, \lambda_{2}, \lambda_{3} \in\{-1,1\}$.

(e) $A=\operatorname{diag}\left(\lambda_{1}, \lambda_{2}\right), B=\operatorname{diag}\left(-\lambda_{1}, 0\right), \lambda_{1}, \lambda_{2} \in\{-1,1\}$.

(f) $A=\operatorname{diag}\left(-\lambda_{1}, 0\right), B=\operatorname{diag}\left(\lambda_{1}, \lambda_{2}\right), \lambda_{1}, \lambda_{2} \in\{-1,1\}$.

(g) $A, B \in\{-1,1\}$.

Now we are going to study two particular cases when the involved matrices do not commute: In order to deal with such situations, we shall use the called CS-decomposition which is now established (see e.g. $[7,10,11,13,15]$ ).

Lemma 2. (CS decomposition) Let $P, Q \in \mathbb{C}_{n, n}$ be two orthogonal projectors. Then there exists $U \in \mathbb{C}_{n}^{\mathrm{U}}$ such that

$$
P=U\left(\begin{array}{cccccc}
I & & & & & \\
& 0 & & & & \\
& & I & & & \\
& & I & & \\
& & & 0 & \\
& & & & 0
\end{array}\right) U^{*}, \quad Q=U\left(\begin{array}{cccccc}
C^{2} & C S & & & \\
C S & S^{2} & & & \\
& & I & & & \\
& & & 0 & & \\
& & & I & \\
& & & & 0
\end{array}\right) U^{*},
$$


where $C, S$ are positive diagonal real matrices satisfying $C^{2}+S^{2}=I$ and the corresponding blocks in the two projection matrices are of the same size.

Theorem 5. Let $A, B \in \mathbb{C}_{n}^{\mathrm{Gl}}$ such that $A B \neq B A$ and $A^{2} B^{2}=B^{2} A^{2}$. Let $a, b \in \mathbb{C} \backslash\{0\}$. If $a A+b B \in \mathbb{C}_{n}^{\mathrm{GI}}$, then one of the following set of disjoint conditions hold

(a) $A^{2}=B^{2}$

(a.1) $a=b$ and $\left(1-3 a^{2}\right)(A+B)=a^{2}(A B A+B A B)$.

(a.2) $a=-b$ and $\left(1-3 a^{2}\right)(A-B)=a^{2}(B A B-A B A)$.

(a.3) $a \neq \pm b, a b(A B A+B)=\left(1-a^{2}-b^{2}\right) A$, and $a b(B A B+A)=\left(1-a^{2}-b^{2}\right) B$.

(b) $A^{2} \neq B^{2}, A^{2} B^{2}=B^{2}$,

- $\left(a^{2}+b^{2}-1\right) B^{2} A\left(I-B^{2}\right)+a b B^{2} A B A\left(I-B^{2}\right)=0$,

- $\left(a^{2}+b^{2}-1\right)\left(I-B^{2}\right) A B^{2}+a b\left(I-B^{2}\right) A B A B^{2}=0$, and

- $\left(a^{2}-1\right)\left(I-B^{2}\right) A\left(I-B^{2}\right)+a b\left(I-B^{2}\right) A B A\left(I-B^{2}\right)=0$.

(c) $A^{2} \neq B^{2}, A^{2} B^{2}=A^{2}$,

- $\left(a^{2}+b^{2}-1\right) A^{2} B\left(I-A^{2}\right)+a b A^{2} B A B\left(I-A^{2}\right)=0$,

- $\left(a^{2}+b^{2}-1\right)\left(I-A^{2}\right) B A^{2}+a b\left(I-A^{2}\right) B A B A^{2}=0$, and

- $\left(b^{2}-1\right)\left(I-A^{2}\right) B\left(I-A^{2}\right)+a b\left(I-A^{2}\right) B A B\left(I-A^{2}\right)=0$.

(d) $A^{2} \neq B^{2}, A^{2} B^{2} \neq A^{2}, A^{2} B^{2} \neq B^{2}$,

- $\left(a^{2}+b^{2}-1\right) B^{2} A\left(I-B^{2}\right)+a b B^{2} A B A\left(I-B^{2}\right)=0$,

- $\left(a^{2}+b^{2}-1\right)\left(I-B^{2}\right) A B^{2}+a b\left(I-B^{2}\right) A B A B^{2}=0$,

- $\left(a^{2}-1\right)\left(I-B^{2}\right) A\left(I-B^{2}\right)+a b\left(I-B^{2}\right) A B A\left(I-B^{2}\right)=0$,

- $\left(a^{2}+b^{2}-1\right) A^{2} B\left(I-A^{2}\right)+a b A^{2} B A B\left(I-A^{2}\right)=0$,

- $\left(a^{2}+b^{2}-1\right)\left(I-A^{2}\right) B A^{2}+a b\left(I-A^{2}\right) B A B A^{2}=0$, and

- $\left(b^{2}-1\right)\left(I-A^{2}\right) B\left(I-A^{2}\right)+a b\left(I-A^{2}\right) B A B\left(I-A^{2}\right)=0$.

Proof. Let us define the orthogonal projectors $P=A^{2}, Q=B^{2}$. By using Lemma 2 and $P Q=Q P$, there exists $S \in \mathbb{C}_{n}^{\mathrm{U}}$ such that

$$
P=A^{2}=S\left(\begin{array}{cccc}
I & 0 & 0 & 0 \\
0 & I & 0 & 0 \\
0 & 0 & 0 & 0 \\
0 & 0 & 0 & 0
\end{array}\right) S^{*}, \quad Q=B^{2}=S\left(\begin{array}{cccc}
I & 0 & 0 & 0 \\
0 & 0 & 0 & 0 \\
0 & 0 & I & 0 \\
0 & 0 & 0 & 0
\end{array}\right) S^{*}
$$

being the corresponding blocks in $P$ and $Q$ of the same size. Let us remark that some blocks in (4) may be absent; but observe that the $i$-th row appears if and only if the $i$-th column appears. We represent matrices $A$ and $B$ as

$$
A=S\left(\begin{array}{cccc}
A_{11} & A_{12} & A_{13} & A_{14} \\
A_{21} & A_{22} & A_{23} & A_{24} \\
A_{31} & A_{32} & A_{33} & A_{34} \\
A_{41} & A_{42} & A_{43} & A_{44}
\end{array}\right) S^{*}, \quad B=S\left(\begin{array}{cccc}
B_{11} & B_{12} & B_{13} & B_{14} \\
B_{21} & B_{22} & B_{23} & B_{24} \\
B_{31} & B_{32} & B_{33} & B_{34} \\
B_{41} & B_{42} & B_{43} & B_{44}
\end{array}\right) S^{*}
$$


From $A=A P$ we get

$$
\left(\begin{array}{cccc}
A_{11} & A_{12} & A_{13} & A_{14} \\
A_{21} & A_{22} & A_{23} & A_{24} \\
A_{31} & A_{32} & A_{33} & A_{34} \\
A_{41} & A_{42} & A_{43} & A_{44}
\end{array}\right)=\left(\begin{array}{cccc}
A_{11} & A_{12} & A_{13} & A_{14} \\
A_{21} & A_{22} & A_{23} & A_{24} \\
A_{31} & A_{32} & A_{33} & A_{34} \\
A_{41} & A_{42} & A_{43} & A_{44}
\end{array}\right)\left(\begin{array}{cccc}
I & 0 & 0 & 0 \\
0 & I & 0 & 0 \\
0 & 0 & 0 & 0 \\
0 & 0 & 0 & 0
\end{array}\right) .
$$

Hence blocks $A_{13}, A_{23}, A_{33}, A_{43}, A_{14}, A_{24}, A_{34}, A_{44}$ are zero. Now, the equality $A=P A$ yields that the blocks $A_{31}, A_{32}, A_{41}, A_{42}$ are also zero. Thus, $A$ can be written as

$$
A=S\left(\begin{array}{cccc}
A_{11} & A_{12} & 0 & 0 \\
A_{21} & A_{22} & 0 & 0 \\
0 & 0 & 0 & 0 \\
0 & 0 & 0 & 0
\end{array}\right) S^{*}
$$

In a similar way we get

$$
B=S\left(\begin{array}{cccc}
B_{11} & 0 & B_{13} & 0 \\
0 & 0 & 0 & 0 \\
B_{31} & 0 & B_{33} & 0 \\
0 & 0 & 0 & 0
\end{array}\right) S^{*}
$$

Since $a A+b B \in \mathbb{C}_{n}^{\mathrm{GI}}$ we get $(a A+b B)^{3}=a A+b B$, which (using $A^{3}=A$ and $B^{3}=B$ ) reduces to

$$
\left(a^{3}-a\right) A+\left(b^{3}-b\right) B+a^{2} b\left(A^{2} B+A B A+B A^{2}\right)+a b^{2}\left(A B^{2}+B A B+B^{2} A\right)=0 .
$$

Using (4) and (6) yields

$$
\begin{array}{rl}
A^{2} B & =S\left(\begin{array}{cccc}
B_{11} & 0 & B_{13} & 0 \\
0 & 0 & 0 & 0 \\
0 & 0 & 0 & 0 \\
0 & 0 & 0 & 0
\end{array}\right) S^{*}, \\
A B A & =S\left(\begin{array}{cccc}
A_{11} B_{11} A_{11} & A_{11} B_{11} A_{12} & 0 & 0 \\
A_{21} B_{11} A_{11} & A_{21} B_{11} A_{12} & 0 & 0 \\
0 & 0 & 0 & 0 \\
0 & 0 & 0 & 0
\end{array}\right) S^{*}, \\
B A^{2} & =S\left(\begin{array}{cccc}
B_{11} & 0 & 0 & 0 \\
0 & 0 & 0 & 0 \\
B_{31} & 0 & 0 & 0 \\
0 & 0 & 0 & 0
\end{array}\right) S^{*}, \\
A B^{2} & =S\left(\begin{array}{cccc}
A_{11} & 0 & 0 & 0 \\
A_{21} & 0 & 0 & 0 \\
0 & 0 & 0 & 0 \\
0 & 0 & 0 & 0
\end{array}\right) S^{*}, \\
B A & S\left(\begin{array}{cccc}
B_{11} A_{11} B_{11} & 0 & B_{11} A_{11} B_{13} & 0 \\
0 & 0 & 0 & 0 \\
B_{31} A_{11} B_{11} & 0 & B_{31} A_{11} B_{33} & 0 \\
0 & 0 & 0 & 0
\end{array}\right) S^{*},
\end{array}
$$




$$
B^{2} A=S\left(\begin{array}{cccc}
A_{11} & A_{12} & 0 & 0 \\
0 & 0 & 0 & 0 \\
0 & 0 & 0 & 0 \\
0 & 0 & 0 & 0
\end{array}\right) S^{*}
$$

Assume that $A^{2} B^{2}=0$. Premultiplying by $A$ and postmultiplying by $B$ we get $A B=0$. Since $B^{2} A^{2}=A^{2} B^{2}=0$ and premultiplying by $B$ and postmultiplying by $A$ we get $B A=0$. Therefore, $A B=B A$, which contradicts one assumption of the Theorem. Thus $A^{2} B^{2} \neq 0$. Hence the first row in (4) must appear.

The proof is split by considering whether the second or third rows in (4) are present.

Assume that second and third rows in (4) are absent. Hence $A^{2}=B^{2}$. Under this assumption, equality (7) is equivalent to

$$
\left(a^{3}+2 a b^{2}-a\right) A+\left(b^{3}+2 a^{2} b-b\right) B+a^{2} b A B A+a b^{2} B A B=0 .
$$

If $a=b$, then (8) reduces to $\left(3 a^{2}-1\right)(A+B)+a^{2}(A B A+B A B)=0$, which is case (a.1). If $a=-b$, then (8) reduces to $\left(3 a^{2}-1\right)(A-B)-a^{2}(A B A-B A B)=0$, which is case (a.2). We suppose that $a^{2} \neq b^{2}$. Premultiplying (8) by $A$, postmultiplying (8) by $B$, and by using $A^{2}=B^{2}, A^{3}=A$, and $B^{3}=B$, equality (8) becomes

$$
\left(a^{3}+2 a b^{2}-a\right) B+\left(b^{3}+2 a^{2} b-b\right) A+a^{2} b B A B+a b^{2} A B A=0 .
$$

Denoting $\alpha=a^{3}+2 a b^{2}-a$ and $\beta=b^{3}+2 a^{2} b-b$, equations (8) and (9) can be written

$$
\left(\begin{array}{cc}
\alpha & \beta \\
\beta & \alpha
\end{array}\right)\left(\begin{array}{c}
A \\
B
\end{array}\right)+a b\left(\begin{array}{cc}
a I & b I \\
b I & a I
\end{array}\right)\left(\begin{array}{c}
A B A \\
B A B
\end{array}\right)=\left(\begin{array}{l}
0 \\
0
\end{array}\right) .
$$

It is evident that

$$
\left(\begin{array}{cc}
a I & -b I \\
-b I & a I
\end{array}\right)\left(\begin{array}{cc}
a I & b I \\
b I & a I
\end{array}\right)=\left(a^{2}-b^{2}\right)\left(\begin{array}{cc}
I & 0 \\
0 & I
\end{array}\right) .
$$

Hence

$$
\begin{aligned}
a b\left(\begin{array}{c}
A B A \\
B A B
\end{array}\right) & =\frac{-1}{a^{2}-b^{2}}\left(\begin{array}{cc}
a I & -b I \\
-b I & a I
\end{array}\right)\left(\begin{array}{c}
\alpha A+\beta B \\
\alpha B+\beta A
\end{array}\right) \\
& =\frac{-1}{a^{2}-b^{2}}\left(\begin{array}{c}
(a \alpha-b \beta) A+(a \beta-b \alpha) B \\
(a \beta-b \alpha) A+(a \alpha-b \beta) B
\end{array}\right) .
\end{aligned}
$$

Making obvious simplifications we get $a \alpha-b \beta=\left(a^{2}+b^{2}-1\right)\left(a^{2}-b^{2}\right)$ and $a \beta-b \alpha=$ $a b\left(a^{2}-b^{2}\right)$. Thus, we arrive at case (a.3).

Assume that second row in (4) is present and third row in (4) is absent. Hence $A^{2} \neq B^{2}$ and $A^{2} B^{2}=B^{2}$. Postmultiplying and premultiplying by $B$ and having in mind that $A^{2} B^{2}=$ $B^{2} A^{2}, B^{3}=B$ we have

$$
A^{2} B=B=B A^{2} .
$$


Let us mention that all matrices appearing in (7) have been written as block matrices. Recall that the first row in (4) must appear and we are under the assumption that the second row in (4) is present and the third row in (4) is absent. Hence (7) is an equality of $2 \times 2$ or $3 \times 3$ block matrices depending whether the fourth row appears or not, respectively. Independently if the fourth block is present or not, looking at representations (5) and (6) we get that block $(1,2)$ of $A$ and $B$ are $A_{12}$ and 0 , respectively. We can easily obtain the block $(1,2)$ of the remaining matrices appearing in $(7)$. By equaling block $(1,2)$ of the left side of $(7)$ to 0 we get

$$
\left(a^{2}+b^{2}-1\right) A_{12}+a b A_{11} B_{11} A_{12}=0,
$$

which, in view of (4), is equivalent to

$$
B^{2}\left[\left(a^{2}+b^{2}-1\right) A+a b A B A\right]\left(A^{2}-B^{2}\right)=0 .
$$

Using (10), identity (12) reduces to

$$
\left(a^{2}+b^{2}-1\right) B^{2} A\left(I-B^{2}\right)+a b B^{2} A B A\left(I-B^{2}\right)=0 .
$$

By equaling the block $(2,1)$ of the left side of $(7)$ to 0 and using the same argument as in the former paragraph, we get

$$
\left(a^{2}+b^{2}-1\right)\left(I-B^{2}\right) A B^{2}+a b\left(I-B^{2}\right) A B A B^{2}=0 .
$$

Again, by equaling the block $(2,2)$ of the left side of $(7)$ to 0 we get $\left(a^{2}-1\right) A_{22}+$ $a b A_{21} B_{11} A_{12}=0$, which is equivalent to

$$
\left(a^{2}-1\right)\left(A^{2}-B^{2}\right) A\left(A^{2}-B^{2}\right)+a b\left(A^{2}-B^{2}\right) A B A\left(A^{2}-B^{2}\right)=0 .
$$

Using $A^{3}=A$ and obvious simplifications lead to $\left(A^{2}-B^{2}\right) A\left(A^{2}-B^{2}\right)=\left(I-B^{2}\right) A\left(I-B^{2}\right)$ and $\left(A^{2}-B^{2}\right) A B A\left(A^{2}-B^{2}\right)=\left(I-B^{2}\right) A B A\left(I-B^{2}\right)$. Therefore, (13) reduces to

$$
\left(a^{2}-1\right)\left(I-B^{2}\right) A\left(I-B^{2}\right)+a b\left(I-B^{2}\right) A B A\left(I-B^{2}\right)=0 .
$$

If the second row in (4) is absent and third row in (4) is present, the reasoning is the same as in the previous paragraph, but interchanging the roles of $A, B$ and $a, b$ obtaining case (c).

Assume that second and third rows in (4) are present. Identity (11), which is valid under the assumption of this paragraph, is equivalent to

$$
A^{2} B^{2}\left[\left(a^{2}+b^{2}-1\right) A+a b A B A\right] A^{2}\left(I-B^{2}\right)=0,
$$

which easily leads to $\left(a^{2}+b^{2}-1\right) B^{2} A\left(I-B^{2}\right)+a b B^{2} A B A\left(I-B^{2}\right)=0$. In a similar procedure as in the previous paragraphs we obtain the remaining equalities of case (d). The proof is completed.

Note. It is easy to see that if $A, B \in \mathbb{C}_{n}^{\mathrm{Gl}}$ satisfy $A^{2}=B^{2}$ and $a, b \in \mathbb{C}$ are such that $a b(A B A+B)=\left(1-a^{2}-b^{2}\right) A$ and $a b(B A B+A)=\left(1-a^{2}-b^{2}\right) B$, then $(a A+b B)^{3}=a A+b B$. 
Hence the conditions given in (a.3) of Theorem 5 combined with the hermitianess of $(a A+b B)^{2}$ are equivalent to $a A+b B \in \mathbb{C}_{n}^{\mathrm{Gl}}$. The same can be said about conditions (a.1) and (a.2).

Note. Observe that if two given matrices $A, B \in \mathbb{C}_{n}^{\mathrm{GI}}$ satisfy one of the conditions (b), (c), or (d), then we can find two scalars $\lambda=\lambda(a, b), \mu=\mu(a, b)$ and four polynomials $p, q, r, s$ in two non-commuting variables such that $p(A, B)=\lambda q(A, B)$ and $r(A, B)=\mu s(A, B)$. By knowing the scalars $\lambda$ and $\mu$, we can find all possible values of $a, b$ by solving a nonlinear $2 \times 2$ system. And after, we can check whether $(a A+b B)^{3}=a A+b B$ and $a A+b B$ is Hermitian.

Therefore, although Theorem 5 does not present an equivalence, this result gives us a procedure of checking if for two given matrices $A, B \in \mathbb{C}_{n}^{\mathrm{Gl}}$, there exist $a, b \in \mathbb{C} \backslash\{0\}$ such that $a A+b B \in \mathbb{C}_{n}^{\mathrm{GI}}$ (and if these scalars exist, then we can find them).

We are going to give of concrete matrices and scalars satisfying any of the conditions of former Theorem 5. Let

$$
X=\left[\begin{array}{cc}
1 & 0 \\
0 & -1
\end{array}\right], \quad Y=\left[\begin{array}{cc}
1 & 0 \\
1 & -1
\end{array}\right] .
$$

After making trivial computations we get

$$
X Y \neq Y X, \quad X^{2}=Y^{2}=I, \quad X Y X=2 X-Y, \quad \text { and } \quad Y X Y=2 Y-X .
$$

(a.1) $A=X, B=Y, a=b=1 / 2$.

(a.2) $A=X, B=-Y, a=-b=1 / 2$.

(a.3) $A=X, B=Y, a+b=1, a \neq \pm b$.

(b) Let us take $A=Y$,

$$
B=\left[\begin{array}{ll}
1 & 0 \\
0 & 0
\end{array}\right]
$$

and $a=1, b=-1$. Obviously, $A B \neq B A$ and $A^{2} B^{2}=B^{2}$. It can be verified that $A, B, a$, and $b$ satisfies all conditions of item (b) of Theorem 5 .

(c) Let us take

$$
A=\left[\begin{array}{ll}
1 & 0 \\
0 & 0
\end{array}\right]
$$

$B=Y, a=-1$, and $b=1$. We have $A B \neq B A$ and $A^{2} B^{2}=A^{2}$. It can be verified that $A, B, a$, and $b$ satisfies all conditions of item (c) of Theorem 5 .

(d) Let

$$
A=\left[\begin{array}{ccc}
1 & 0 & 0 \\
1 & -1 & 0 \\
0 & 0 & 0
\end{array}\right], \quad B=\left[\begin{array}{ccc}
1 & 0 & 0 \\
0 & 0 & 0 \\
1 & 0 & -1
\end{array}\right],
$$

$a=1, b=-1$. We have $A B \neq B A, A^{2} B^{2} \neq A^{2}, A^{2} B^{2} \neq B^{2}$, and $A^{2} B^{2}=B^{2} A^{2}$. It can be verified that $A, B, a$, and $b$ satisfies all six conditions of item (d) of Theorem 5 . 
Theorem 6. Let $A, B \in \mathbb{C}_{n}^{\mathrm{Gl}}$ such that $A^{2} B^{2}-B^{2} A^{2}$ is nonsingular and $a, b \in \mathbb{C} \backslash\{0\}$. Then $a A+b B \in \mathbb{C}_{n}^{\mathrm{Gl}}$ if and only if $a^{2}, b^{2} \in \mathbb{R}$ and

$$
a b B A B=\left(1-b^{2}\right) B, \quad a b A B A=\left(1-a^{2}\right) A, \quad a A^{2} B+b A B^{2}=0, \quad a B A^{2}+b B^{2} A=0 .
$$

Proof. In the first part of the proof, we will represent matrices $A$ and $B$ in a convenient form. Let us define the orthogonal projectors $P=A^{2}$ and $Q=B^{2}$. Now, the CS decomposition is going to be used for $P$ and $Q$. Taking into account that $A^{2} B^{2}-B^{2} A^{2}$ is nonsingular, we can write

$$
A^{2}=P=U\left(\begin{array}{cc}
I & 0 \\
0 & 0
\end{array}\right) U^{*}, \quad B^{2}=Q=U\left(\begin{array}{cc}
C^{2} & C S \\
C S & S^{2}
\end{array}\right) U^{*}
$$

where $U, C$, and $S$ have the same meaning as in Lemma 2. We represent matrices $A$ and $B$ as

$$
A=U\left(\begin{array}{cc}
A_{1} & A_{2} \\
A_{3} & A_{4}
\end{array}\right) U^{*}, \quad B=U\left(\begin{array}{cc}
B_{1} & B_{2} \\
B_{3} & B_{4}
\end{array}\right) U^{*}
$$

being the size of each block the same as the corresponding one in (15). From $A=A P$ we get

$$
\left(\begin{array}{ll}
A_{1} & A_{2} \\
A_{3} & A_{4}
\end{array}\right)=\left(\begin{array}{cc}
A_{1} & A_{2} \\
A_{3} & A_{4}
\end{array}\right)\left(\begin{array}{cc}
I & 0 \\
0 & 0
\end{array}\right)=\left(\begin{array}{ll}
A_{1} & 0 \\
A_{3} & 0
\end{array}\right)
$$

Hence $A_{2}=0$ and $A_{4}=0$. Now, the equality $A=P A$ yields $A_{3}=0$. Thus,

$$
A=U\left(A_{1} \oplus 0\right) U^{*} .
$$

Since $A^{2}=P$, we arrive at $A_{1}^{2}=I$. Now, from $B=B Q$ we get

$$
\left(\begin{array}{ll}
B_{1} & B_{2} \\
B_{3} & B_{4}
\end{array}\right)=\left(\begin{array}{cc}
B_{1} & B_{2} \\
B_{3} & B_{4}
\end{array}\right)\left(\begin{array}{cc}
C^{2} & C S \\
C S & S^{2}
\end{array}\right)
$$

Therefore

$$
B_{1}=B_{1} C^{2}+B_{2} C S, \quad B_{2}=B_{1} C S+B_{2} S^{2}, \quad B_{3}=B_{3} C^{2}+B_{4} C S, \quad B_{4}=B_{3} C S+B_{4} S^{2} .
$$

Taking into account that $C^{2}+S^{2}=I, C S=S C$, and $C, S$ are nonsingular, the four relations of (17) reduce to

$$
B_{2} C=B_{1} S, \quad B_{4} C=B_{3} S .
$$

Analogously, from $B=Q B$ we have

$$
C B_{3}=S B_{1}, \quad C B_{4}=S B_{2} .
$$

Let us define $T=S C^{-1}$. It is easy to deduce that (18) and (19) are equivalent to

$$
B_{2}=B_{1} T, \quad B_{3}=T B_{1}, \quad B_{4}=T B_{1} T .
$$

Hence

$$
B=U\left(\begin{array}{cc}
B_{1} & B_{1} T \\
T B_{1} & T B_{1} T
\end{array}\right) U^{*}
$$


In the second part of the proof, we suppose that $a A+b B \in \mathbb{C}_{n}^{\mathrm{Gl}}$. Recall that a matrix $M \in \mathbb{C}_{n, n}$ satisfies $M \in \mathbb{C}_{n}^{\mathrm{Gl}}$ if and only if $M=M^{3}$ and $M^{2}$ is Hermitian. Taking into account that $A^{3}=A, B^{3}=B$, and $(a A+b B)^{3}=a A+b B$, we get

$$
\left(a^{3}-a\right) A+a^{2} b\left(A^{2} B+A B A+B A^{2}\right)+a b^{2}\left(A B^{2}+B A B+B^{2} A\right)+\left(b^{3}-b\right) B=0 .
$$

From (16) and (20) we get

$$
\begin{aligned}
A^{2} B & =U\left(\begin{array}{cc}
B_{1} & B_{1} T \\
0 & 0
\end{array}\right) U^{*} \\
A B A & =U\left(\begin{array}{cc}
A_{1} B_{1} A_{1} & 0 \\
0 & 0
\end{array}\right) U^{*} \\
B A^{2} & =U\left(\begin{array}{cc}
B_{1} & 0 \\
T B_{1} & 0
\end{array}\right) U^{*} \\
A B^{2} & =U\left(\begin{array}{cc}
A_{1} C^{2} & A_{1} C S \\
0 & 0
\end{array}\right) U^{*} \\
B A B & =U\left(\begin{array}{cc}
B_{1} A_{1} B_{1} & B_{1} A_{1} B_{1} T \\
T B_{1} A_{1} B_{1} & T B_{1} A_{1} B_{1} T
\end{array}\right) U^{*} \\
B^{2} A & =U\left(\begin{array}{cc}
C^{2} A_{1} & 0 \\
C S A_{1} & 0
\end{array}\right) U^{*} .
\end{aligned}
$$

Observe that in view of representations (16), (20), and (22)-(26), all matrices involved in (21) are written as $2 \times 2$ block matrices. Hence, equality (21) can be viewed as an equality of two $2 \times 2$ block matrices. From the block $(2,2)$ of the relation (21) we get $a b^{2} T B_{1} A_{1} B_{1} T+$ $\left(b^{3}-b\right) T B_{1} T=0$, which reduces to

$$
a b B_{1} A_{1} B_{1}=\left(1-b^{2}\right) B_{1} .
$$

In view of the representations (20) and (25), the above equality (27) implies

$$
a b B A B=\left(1-b^{2}\right) B .
$$

By a reasoning inverting the roles of $A$ and $B$ we have

$$
a b A B A=\left(1-a^{2}\right) A .
$$

Substituting (29) and (28) into (21) we obtain

$$
a\left(A^{2} B+B A^{2}\right)+b\left(A B^{2}+B^{2} A\right)=0 .
$$

Analogously as we pointed before, the equality (30) can be viewed as an equality of two $2 \times 2$ block matrices. From the block $(1,2)$ of relation (30) and by considering representations (22), (23), (24), and (26) we get

$$
a B_{1} T+b A_{1} C S=0 .
$$

Recall that $T=S C^{-1}, S C=C S$, and $S$ is nonsingular. Thus, (31) can be written also as

$$
a B_{1}+b A_{1} C^{2}=0 .
$$


From representations (22), (24), and equations (31), (32) we get

$$
a A^{2} B+b A B^{2}=U\left[a\left(\begin{array}{cc}
B_{1} & B_{1} T \\
0 & 0
\end{array}\right)+b\left(\begin{array}{cc}
A_{1} C^{2} & A_{1} C S \\
0 & 0
\end{array}\right)\right] U^{*}=0 .
$$

Using $a A^{2} B+b A B^{2}=0$ and (30) we get $a B A^{2}+b B^{2} A=0$.

Premultiplying $a A^{2} B+b A B^{2}=0$ by $A$ we get $a A B+b A^{2} B^{2}=0$, and using representations (15), (16), and (20), we get $a A_{1} B_{1}+b C^{2}=0$. Postmultimplying $a B A^{2}+b B^{2} A=0$ by $A$ and using representations (15), (16), and (20) we get $a B_{1} A_{1}+b C^{2}=0$. Therefore,

$$
A_{1} B_{1}=B_{1} A_{1}=-\frac{b}{a} C^{2} .
$$

Now, we shall use that $(a A+b B)^{2}$ is Hermitian. From (33) we get

$$
\begin{aligned}
A B+B A & =U\left[\left(\begin{array}{cc}
A_{1} & 0 \\
0 & 0
\end{array}\right)\left(\begin{array}{cc}
B_{1} & B_{1} T \\
T B_{1} & T B_{1} T
\end{array}\right)+\left(\begin{array}{cc}
B_{1} & B_{1} T \\
T B_{1} & T B_{1} T
\end{array}\right)\left(\begin{array}{cc}
A_{1} & 0 \\
0 & 0
\end{array}\right)\right] U^{*} \\
& =U\left[-\frac{b}{a}\left(\begin{array}{cc}
2 C^{2} & C S \\
C S & 0
\end{array}\right)\right] U^{*},
\end{aligned}
$$

hence

$$
\begin{aligned}
(a A+b B)^{2} & =a^{2} A^{2}+b^{2} B^{2}+a b(A B+B A) \\
& =U\left[a^{2}\left(\begin{array}{cc}
I & 0 \\
0 & 0
\end{array}\right)+b^{2}\left(\begin{array}{cc}
C^{2} & C S \\
C S & S^{2}
\end{array}\right)-b^{2}\left(\begin{array}{cc}
2 C^{2} & C S \\
C S & 0
\end{array}\right)\right] U^{*} \\
& =U\left(\begin{array}{cc}
a^{2} I-b^{2} C^{2} & 0 \\
0 & b^{2} S^{2}
\end{array}\right) U^{*}
\end{aligned}
$$

The $(2,2)$ block of $(a A+b B)^{2}$ inherits the hermitianess property, hence $b^{2} S^{2}$ is Hermitian, and by recalling that $S$ is a diagonal and real matrix, we deduce that $b^{2} \in \mathbb{R}$. By interchanging the roles of $a$ and $b$, we can conclude that $a^{2} \in \mathbb{R}$.

In the third and last part of the proof, we suppose that $a^{2}, b^{2} \in \mathbb{R}$ and the relations (14) are satisfied. We must prove that $(a A+b B)^{3}=a A+b B$ and $(a A+b B)^{2}$ is Hermitian:

$$
\begin{aligned}
(a A & +b B)^{3}-(a A+b B)= \\
& =\left(a^{3}-a\right) A+a^{2} b\left(A^{2} B+A B A+B A^{2}\right)+a b^{2}\left(A B^{2}+B A B+B^{2} A\right)+\left(b^{3}-b\right) B \\
& =a^{2} b\left(A^{2} B+B A^{2}\right)+a b^{2}\left(A B^{2}+B^{2} A\right) \\
& =a b\left(a A^{2} B+b A B^{2}\right)+a b\left(a B A^{2}+b B^{2} A\right)=0 .
\end{aligned}
$$

Now, we shall prove that $(a A+b B)^{2}$ is Hermitian. Since $a^{2}, b^{2} \in \mathbb{R}$, the matrices $A^{2}$ and $B^{2}$ are Hermitian, and $(a A+b B)^{2}=a^{2} A^{2}+a b(A B+B A)+b^{2} B^{2}$, it is sufficient to prove that $a b(A B+B A)$ is Hermitian. Premultiplying the third relation of (14) by $A$ we get $a A B+b A^{2} B^{2}=0$ and postmultiplying the fourth relation by $A$ we obtain $a B A+b B^{2} A^{2}=0$. Thus

$$
a b(A B+B A)=a b\left(-\frac{b}{a} A^{2} B^{2}-\frac{b}{a} B^{2} A^{2}\right)=-b^{2}\left(A^{2} B^{2}+B^{2} A^{2}\right)=-b^{2}(P Q+Q P) .
$$

Recall that $b^{2} \in \mathbb{R}$ and $P, Q$ are both Hermitian, and therefore, $-b^{2}(P Q+Q P)$ is Hermitian. The proof is completed. 
Next example shows that the set of matrices and scalars satisfying Theorem 6 is not empty. Let $t \in] 0,+\infty[$ and let us define

$$
A=\left[\begin{array}{ll}
1 & 0 \\
0 & 0
\end{array}\right], \quad X=\left[\begin{array}{cc}
1 & t \\
t & t^{2}
\end{array}\right], \quad B=\frac{1}{1+t^{2}} X .
$$

Trivially we have $A=A^{\dagger}$ because $A$ is an orthogonal projector. Since we have $X^{2}=\left(1+t^{2}\right) X$ we get $B^{3}=B$. Moreover, $B^{2}$ is Hermitian because $X$ is Hermitian. Thus $B=B^{\dagger}$. The following equalities can be trivially obtained: $A^{2} B=A B^{2} \neq 0, B A^{2}=B^{2} A \neq 0$,

$$
A^{2} B^{2}-B^{2} A^{2}=\frac{1}{1+t^{2}}\left[\begin{array}{cc}
0 & t \\
-t & 0
\end{array}\right], \quad B A B=\frac{1}{1+t^{2}} B, \quad A B A=\frac{1}{1+t^{2}} A .
$$

Thus, if there exist nonzero scalars $a, b$ such that

$$
\frac{1-b^{2}}{a b}=\frac{1}{1+t^{2}}, \quad \frac{1-a^{2}}{a b}=\frac{1}{1+t^{2}}, \quad a+b=0, \quad a^{2}, b^{2} \in \mathbb{R},
$$

then this example will show that the set of matrices and scalars satisfying Theorem 6 is not empty. But, if $a, b$ satisfy (34), then $a= \pm \sqrt{1+t^{2}} / t, b=\mp \sqrt{1+t^{2}} / t$.

Now we are ready to solve the following problem:

Problem: Let $A, B \in \mathbb{C}_{n}^{\mathrm{Gl}}$ be two given noncommuting matrices. Let us denote by $\Pi$ the orthogonal projector onto the null space of $A^{2} B^{2}-B^{2} A^{2}$. If $\Pi A=A \Pi$ and $\Pi B=B \Pi$, we can answer the following two questions:

(i) Are there $a, b \in \mathbb{C} \backslash\{0\}$ such that $a A+b B \in \mathbb{C}_{n}^{\mathrm{Gl}}$ ?

(ii) If the answer to Question (i) is affirmative, find such scalars $a$ and $b$.

Let us denote $P=A^{2}$ and $Q=B^{2}$. By Lemma 2 there exists $U \in \mathbb{C}_{n}^{U}$ such that

$$
P=U\left(\begin{array}{cc|cccc}
I & & & & & \\
& 0 & & & & \\
\hline & & I & & & \\
& & & I & & \\
& & & 0 & \\
& & & & 0
\end{array}\right) U^{*}=U\left(R_{P} \oplus T_{P}\right) U^{*},
$$

and

$$
Q=U\left(\begin{array}{cc|cccc}
C^{2} & C S & & & & \\
C S & S^{2} & & & & \\
\hline & & I & & & \\
& & & 0 & & \\
& & & & I & \\
& & & & 0
\end{array}\right) U^{*}=U\left(R_{Q} \oplus T_{Q}\right) U^{*},
$$

where $R_{P}, R_{Q}, T_{P}$, and $T_{Q}$ are square matrices and the size of $R_{P}$ equals to the size of $R_{Q}$ and it is twice the size of $C$. 
Let $\Pi=U(O \oplus I) U^{*}$, where the size of the last identity matrix is the size of $T_{P}$ (and $\left.T_{Q}\right)$. In [6, Pg. 763] it was proved that $\Pi$ is the orthogonal projector onto the null space of $P Q-Q P$. Since $A \Pi=\Pi A$, we can write $A=U\left(A_{1} \oplus A_{2}\right) U^{*}$ for some square matrices $A_{1}, A_{2}$, being the size of $A_{1}$ equals to the size of $R_{P}$. In a similar manner, we can express $B$ as $B=U\left(B_{1} \oplus B_{2}\right) U^{*}$ with $B_{1}$ and $R_{Q}$ having the same size.

It is easy to see the following facts:

(1) $A_{1}, A_{2}, B_{1}, B_{2}$ are generalized involutive matrices.

(2) $a A+b B \in \mathbb{C}_{n}^{\mathrm{Gl}}$ if and only if $a A_{1}+b B_{1}, a A_{2}+b B_{2}$ are generalized involutive matrices.

(3) $A_{1}^{2} B_{1}^{2}=B_{1}^{2} A_{1}^{2}$.

(4) $A_{2}^{2} B_{2}^{2}-B_{2}^{2} A_{2}^{2}$ is nonsingular.

Thus, the proposed problem can be solved by studying simultaneously the problems concerning the linear combinations $a A_{1}+b B_{1}, a A_{2}+b B_{2}$.

Acknowledgment: The authors wish to thank one referee for his/her suggestion to improve the quality and the readability of this paper.

\section{References}

[1] J.K. Baksalary, O.M. Baksalary, and X. Liu, Further properties of generalized and hypergeneralized projectors, Linear Algebra Appl., 389 (2004) 295-303.

[2] J.K. Baksalary, O.M. Baksalary, and H. Özdemir, A note on linear combinations of commuting tripotent matrices, Linear Algebra Appl., 388 (2004) 45-51.

[3] O.M. Baksalary, J. Benítez, On linear combinations of two commuting hypergeneralized projectors, Comput. Math. Appl., 56 (2008) 2481-2489.

[4] R.B. Bapat, Moore-Penrose inverse of set inclusion matrices, Linear Algebra Appl., 318 (2000) 35-44.

[5] J. Benítez, Moore-Penrose inverses and commuting elements of $C^{*}$-algebras, J. Math. Anal. Appl., 345 (2008) 766-770.

[6] J. Benítez and V. Rakočević, Applications of CS decomposition in linear combinations of two orthogonal projectors, Appl. Math. Comput., 203 (2008) 761-769.

[7] A. Björck, Numerical Methods for Least Squares Problems, Society for Industrial and Applied Mathematics (SIAM), Philadelphia, PA, 1996.

[8] E. Boasso, On the Moore-Penrose inverse in $C^{*}$-algebras, Extracta Math., 21 (2006) 93-106.

[9] S.L. Campbell and C.D. Meyer, Jr., Generalized Inverses of Linear Transformations, Pitman, London, 1979. 
[10] J. Dauxois and G.M. Nkiet, Canonical analysis of two Euclidian subspaces and its applications, Linear Algebra Appl., 264 (1997) 355-388.

[11] G. H. Golub and C. F. Van Loan, Matrix Computations, third ed., Johns Hopkins Studies in the Mathematical Sciences, Johns Hopkins University Press, Baltimore, MD, 1996.

[12] J. Groß and G. Trenkler, Generalized and hypergeneralized projectors, Linear Algebra Appl., 264 (1997) 463-474.

[13] M. Hegland, J. Garcke, and V. Challis, The combination technique and some generalisations, Linear Algebra Appl., 420 (2007) 249-275.

[14] C.H. Hung and T.L. Markham, The Moore-Penrose inverse of a sum of matrices, J. Austral. Math. Soc. Ser., A 24 (1977) 385-392.

[15] C. C. Paige and M. Wei, History and generality of the CS decomposition, Linear Algebra Appl., 209 (1994) 303-326.

[16] J.R. Schott, Matrix Analysis for Statistics, John Wiley and Sons, Wiley-Interscience, New York, NY, 1997.

[17] G.A.F. Seber, A Matrix Handbook For Statisticians, John Wiley and Sons, WileyInterscience, Hoboken, New Jersey, 2008.

[18] G.W. Stewart, A note on generalized and hypergeneralized projectors, Linear Algebra Appl., 412 (2006) 408-411.

[19] Trenkler, G. Characterizations of oblique and orthogonal projectors. In T . Caliński and R. Kala (Eds.), Proceedings of the International Conference on Linear Statistical Inference LINSTAT'W, 255-270. Kluwer Academic Publishers, Netherlands (1994). 